\title{
Antimicrobial Nonsusceptibility of Gram-Negative Bloodstream Isolates, Veterans Health Administration System, United States, 2003-2013 ${ }^{1}$
}

\author{
Michihiko Goto, Jennifer S. McDanel, Makoto M. Jones, Daniel J. Livorsi, Michael E. OhI, \\ Brice F. Beck, Kelly K. Richardson, Bruce Alexander, Eli N. Perencevich
}

\begin{abstract}
Bacteremia caused by gram-negative bacteria is associated with serious illness and death, and emergence of antimicrobial drug resistance in these bacteria is a major concern. Using national microbiology and patient data for 2003-2013 from the US Veterans Health Administration, we characterized nonsusceptibility trends of community-acquired, community-onset; healthcare-associated, community-onset; and hospital-onset bacteremia for selected gram-negative bacteria (Escherichia coli, Klebsiella spp., Pseudomonas aeruginosa, and Acinetobacter spp.). For 47,746 episodes of bacteremia, the incidence rate was 6.37 episodes $/ 10,000$ person-years for community-onset bacteremia and $4.53 \mathrm{ep}-$ isodes $/ 10,000$ patient-days for hospital-onset bacteremia. For Klebsiella spp., P. aeruginosa, and Acinetobacter spp., we observed a decreasing proportion of nonsusceptibility across nearly all antimicrobial drug classes for patients with healthcare exposure; trends for community-acquired, community-onset isolates were stable or increasing. The role of infection control and antimicrobial stewardship efforts in inpatient settings in the decrease in drug resistance rates for hospital-onset isolates needs to be determined.
\end{abstract}

$\mathrm{D}$ espite advances in public health and medical care, bacteremia is still a major cause of illness and death (1-3). Bacteremia caused by gram-negative bacteria is a frequent cause of severe sepsis and septic shock $(4,5)$ and poses serious therapeutic challenges. Treatment options are limited because of increased infections with multidrug-

Author affiliations: lowa City Veterans Affairs Health Care System, lowa City, lowa, USA (M. Goto, J.S. McDanel, D.J. Livorsi,

M.E. Ohl, B.F. Beck, K.K. Richardson, B. Alexander,

E.N. Perencevich); University of lowa Carver College of Medicine, lowa City (M. Goto, J.S. McDanel, D.J. Livorsi, M.E. Ohl,

E.N. Perencevich); Salt Lake City Veterans Affairs Health Care System, Salt Lake City, Utah, USA (M.M. Jones); University of Utah School of Medicine, Salt Lake City USA (M.M. Jones)

DOI: https://doi.org/10.32301/eid2311.161214 resistant, gram-negative bacteria in community and hospital settings. Understanding the epidemiology of infections with gram-negative bacteria is needed to improve guidelines providing empiric therapy recommendations and adequately allocate resources toward infection control and antimicrobial stewardship programs.

Descriptions of the epidemiology of gram-negative bacteremia have been limited by care settings, referral bias, small geographic regions, short study duration, or case identification by use of administrative code data $(6-10)$. As modern healthcare systems become increasingly diverse and complex, large-scale studies are needed that accurately estimate the burden of antimicrobial resistance and chronologic trends of gram-negative bacteremia (11-13).

The Veterans Health Administration (VHA) is the largest healthcare system in the United States and has $\approx 8$ million veterans enrolled (14). The VHA uses an integrated electronic health record and a nationwide data repository. Microbiological results from all VHA facilities have been added to the data repository, which enables identification of all cases of gram-negative bacteremia across the entire VHA system. These results enable examination of national trends in gram-negative bacteremia in a geographically dispersed and diverse population.

We previously reported incidence rates of communityonset and hospital-onset bacteremia caused by 3 species of gram-negative bacteria in a cohort of patients admitted to the VHA system during 2003-2013 (15). In this article, we expand on that study by reporting trends in antimicrobial nonsusceptibility (intermediate or resistant) among bacteremia isolates of 4 selected gram-negative bacteria (Escherichia

\footnotetext{
${ }^{1}$ Preliminary results from this study were presented at the 25th European Congress of Clinical Microbiology and Infectious Diseases, April 25-28, 2015, Copenhagen, Denmark; and at the Society for Healthcare Epidemiology of America Spring Meeting, May 14-17, 2015, Orlando, Florida, USA.
} 
coli, Klebsiella spp., Pseudomonas aeruginosa, and Acinetobacter spp.) in the national VHA healthcare system.

\section{Methods}

\section{Study Population}

We analyzed a retrospective cohort of all veterans who were admitted to acute-care units at VHA hospitals during January 2003-December 2013 and who had positive blood cultures for E. coli, Klebsiella spp., P. aeruginosa, or Acinetobacter spp. between 48 hours before admission and time of discharge. We used these 4 bacterial species a priori because studies consistently showed that these organisms represent most gram-negative bacteria causing bacteremia across various healthcare settings $(3,16-22)$. If 1 patient had multiple positive blood cultures for the same species during 1 hospital admission, we included only the first isolate. To estimate the population at risk for community-onset bacteremia, we identified the number of veterans who had visited VHA clinics and emergency departments inside the catchment areas of acute inpatient care facilities by calendar year (outpatient denominator for community-onset bacteremia). To estimate the population at risk for hospital-onset bacteremia, we calculated patientdays in acute inpatient care units (inpatient denominator for hospital-onset bacteremia).

Data for 130 VHA acute-care hospitals in the 48 contiguous states, the District of Columbia, and Puerto Rico were used in this study (23). Using the US Department of Agriculture and Department of Health and Human Services Rural-Urban Commuting Areas System, we found that 107 facilities were located in urban areas, 22 in rural areas, and 1 in a highly rural area (24). These VA hospitals also serve as referral centers for $>1,400$ outreach clinics. Acute inpatient capacities were 10-260 beds. Total acute inpatient capacity was $\approx 10,000$ acute-care beds, including 1,900 authorized intensive care unit beds (25). We did not include stays at mental health, rehabilitation, and nursing home care units. All but 3 included hospitals have on-site microbiology laboratories, and they are required to conduct quality control/quality assessment routinely per requirements of VHA-designated accreditation organizations and to use methods and equipment approved by the Food and Drug Administration (FDA; Silver Spring, MD, USA).

The institutional review board at the University of Iowa and the research and development committee of the Iowa City Veterans Affairs Health Care System approved this study. A waiver of informed consent was issued for this retrospective analysis.

\section{Data Source}

We obtained data through Veterans Affairs Informatics and Computing Infrastructure, which includes data extracted from the VHA integrated electronic medical record system. Susceptibility results in microbiology reports (susceptible/ intermediate/resistant) were recorded in a standardized manner, and isolates were classified as nonsusceptible if they were reported as intermediate resistance or resistant. MICs or sizes of inhibition zones were not typically available.

\section{Definitions}

Bacteremia episodes were classified according to Centers for Diseases Control and Prevention (Atlanta, GA, USA) criteria as community-onset and hospital-onset. This classification was based on time of the first positive blood culture as a standard definition (26).

An episode of bacteremia was considered to be community-onset when the first positive blood culture was collected between 48 hours before and $<48$ hours after admission. For episodes of hospital-onset bacteremia, the first positive blood culture was collected $\geq 48$ hours after admission.

We further categorized community-onset bacteremia episodes as community-acquired, community-onset and healthcare-associated, community-onset on the basis of healthcare exposure in the VHA before hospital admission. Episodes were classified as healthcare-associated, community-onset when the patient had been admitted to an acutecare facility $\leq 90$ days before onset of bacteremia; was a resident of a nursing home or rehabilitation facility; was receiving renal replacement therapy; or received wound care or specialized nursing care in an outpatient setting or at home in the 30 days before onset of bacteremia (19). If the patient with community-onset bacteremia did not meet these criteria, the episode was classified as community-acquired, community-onset. Information for healthcare exposure outside the VHA before admission was available only if the VHA was the payer of care.

We categorized antimicrobial drugs listed on susceptibility reports into antimicrobial classes by using interim standard definitions for acquired resistance produced by an international panel of experts from the Centers for Disease Control and Prevention and the European Centre for Disease Prevention and Control (Solna, Sweden) (27). We provide included antimicrobial agents and antimicrobial classes for each organism (online Technical Appendix Tables 1-3, https://wwwnc.cdc.gov/EID/ article/23/11/16-1214-Techapp1.pdf). Isolates were considered nonsusceptible if they were not susceptible to $\geq 1$ agent in an antimicrobial class (27).

\section{Measurements}

We focused primarily on annual proportions and trends of antimicrobial nonsusceptibility rates for the 4 most clinically relevant antimicrobial classes (carbapenems, extended-spectrum cephalosporins, aminoglycosides, and fluoroquinolones) used in gram-negative bacteremia 
management. We also evaluated incidence rates per 10,000 outpatients for community-onset bacteremia and incidence rates per 10,000 patient-days and per 1,000 admissions for hospital-onset bacteremia for each organism. To enable comparisons with previously reported studies, we calculated age-standardized incidence rates by using a direct method, used US population data for 2000 as a standard population for community-onset bacteremia (28), and reported incidence rates with 2 denominators for hospital-onset bacteremia.

\section{Statistical Analysis}

We summarized characteristics of the study population and incidence rates for all 3 categories by using descriptive statistics. We calculated $95 \%$ CIs by using the Clopper-Pearson exact method (29) for crude incidence rates. Proportions among acquisition categories were compared by using Fisher exact tests. To assess annual trends in proportions of nonsusceptible isolates, we used the CochraneArmitage $\chi^{2}$ test for trend. All $\mathrm{p}$ values were 2-sided, and values $<0.05$ were considered statistically significant. All statistical analyses were performed by using SAS version 9.4 (SAS Institute Inc., Cary, NC, USA).

\section{Results}

\section{Study Population Demographics and Overall Incidence Rates}

During 2003-2013, the VHA provided 53,576,096 personyears of outpatient care and 30,015,733 patient-days of acute inpatient care. We obtained demographic characteristics of the study population (Table 1). A total of 47,746 episodes of gram-negative bacteremia occurred during the study. Most (96.4\%) patients were male, and communityacquired, community-onset cases accounted for $42.3 \%$ of cases of gram-negative bacteremia, followed by healthcareassociated, community-onset (29.2\%) and hospital-onset (28.5\%) (Table 2; online Technical Appendix Figure 1).

Overall incidence rates from the entire study were 3.77 episodes/10,000 person-years for community-acquired, community-onset gram-negative bacteremia, 2.60 episodes/10,000 person-years for healthcare-associated, community-onset gram-negative bacteremia, and 4.53 episodes/10,000 patient-days (2.40 episodes/1,000 admissions) for hospital-onset gram-negative bacteremia.
Age-standardized incidence rates were 1.92 episodes/10,000 person-years for community-acquired, community-onset gram-negative bacteremia and 1.40 episodes/10,000 person-years for healthcare-associated, community-onset gram-negative bacteremia (online Technical Appendix Figures 2-4).

\section{Trends for Antimicrobial Drug Susceptibilities}

Susceptibility data were available for $>96 \%$ of all organism-antimicrobial drug combinations. The only exception was carbapenem susceptibility of Acinetobacter spp., which was available for $90 \%$ of isolates tested.

\section{E. coli}

Carbapenem nonsusceptibility for $E$. coli was infrequent $(0.3 \%)$ (Figure 1). However, nonsusceptibility to carbapenems increased for community-acquired, communityonset isolates ( 0 in $2003-2007$ and $0.2 \%$ in $2008-2013$, $\mathrm{p}<0.01$ by test for trend). Nonsusceptibility for healthcareassociated, community-onset ( $\mathrm{p}=0.92$ by test for trend) and hospital-onset ( $\mathrm{p}=0.82$ by test for trend) isolates did not change.

Overall, $6.3 \%$ of $E$. coli isolates were nonsusceptible to extended-spectrum cephalosporins; an increasing proportion were nonsusceptible (4.1\% in $2003-2007$ and $7.9 \%$ in 2008-2013, $\mathrm{p}<0.01$ by test for trend). Extended-spectrum cephalosporin-nonsusceptible isolates were more frequent for patients with previous or recent healthcare exposure (community-acquired, community-onset 3.9\%; healthcareassociated, community-onset $9.0 \%$, and hospital-onset $9.8 \%, \mathrm{p}<0.01)$. The proportion of isolates that were nonsusceptible to extended-spectrum cephalosporin increased for all categories (community-acquired, community-onset; healthcare-associated, community-onset; hospital-onset, $\mathrm{p}<0.01$ by test for trend).

The overall proportion of isolates that were nonsusceptible to aminoglycosides was $11.9 \%$. Healthcare exposure was associated with higher rates of nonsusceptibility (community-acquired, community-onset 9.0\%; healthcareassociated, community-onset $15.6 \%$; hospital-onset $16.0 \%$, $\mathrm{p}<0.01)$. We observed increasing nonsusceptibility rates during the study (10.5\% in 2003-2007 and $12.9 \%$ in 2008 $2013, p<0.01$ by test for trend).

Incidence rates for fluoroquinolone-nonsusceptible isolates rapidly increased during the first half of the study

\begin{tabular}{lccccccccccccc}
\hline \multicolumn{10}{c}{ Table 1. Characteristics of acute-care patients, by year, Veterans Health Administration System, United States, 2003-2013 } \\
\hline Characteristic & 2003 & 2004 & 2005 & 2006 & 2007 & 2008 & 2009 & 2010 & 2011 & 2012 & 2013 \\
\hline Outpatient care & & & & & & & & & & & \\
$\quad$ No. outpatients & 4,357 & 4,532 & 4,623 & 4,705 & 4,719 & 4,784 & 4,945 & 5,083 & 5,193 & 5,281 & 5,355 \\
$\quad$ Mean age, y, on Jan 1 & 61.5 & 61.7 & 61.7 & 61.8 & 61.6 & 61.4 & 61.1 & 61.0 & 60.9 & 60.7 & 60.6 \\
\hline Inpatient care & & & & & & & & & & & & & \\
$\quad$ No. hospital admissions & 470 & 481 & 489 & 492 & 498 & 516 & 536 & 549 & 552 & 552 & 545 \\
$\quad$ Average length of stay, d & 6.0 & 5.8 & 5.6 & 5.5 & 5.4 & 5.3 & 5.1 & 5.0 & 5.0 & 4.8 & 4.8 \\
$\quad$ No. patient-days & 2,805 & 2,783 & 2,749 & 2,718 & 2,701 & 2,745 & 2,747 & 2,748 & 2,735 & 2,675 & 2,611 \\
\hline
\end{tabular}


Table 2. Characteristics of patients with bacteremia caused by gram-negative bacteria, Veterans Health Administration System, United States, 2003-2013*

\begin{tabular}{lccccc}
\hline Characteristic & Total & Escherichia coli & Klebsiella spp. & $\begin{array}{c}\text { Pseudomonas } \\
\text { aeruginosa }\end{array}$ & Acinetobacter spp. \\
\hline No. & 47,746 & 24,557 & 14,270 & 6,929 & 1,990 \\
Age, y, mean \pm SD & $69.1 \pm 2.2$ & $69.8 \pm 12.3$ & $68.5 \pm 12.1$ & $68.5 \pm 11.9$ & $65.7 \pm 12.6$ \\
\hline Sex, \% & 96.40 & 95.30 & 97.30 & 97.90 & 97.30 \\
M & 3.60 & 4.70 & 2.70 & 2.10 & 2.70 \\
F & & & & \\
Infection & $20,210(42.3)$ & $13,515(55.0)$ & $4,802(33.7)$ & $1,454(21.0)$ & $439(22.1)$ \\
CA-CO & $13,932(29.2)$ & $6,857(27.9)$ & $4,242(29.7)$ & $2,262(32.7)$ & $571(28.7)$ \\
HCA-CO & $13,604(28.5)$ & $4,185(17.0)$ & $5,226(36.6)$ & $3,213(46.4)$ & $980(49.3)$ \\
HO & $3,819(8.0)$ & $838(3.4)$ & $1,389(9.7)$ & $1,174(16.9)$ \\
HO (ICU onset) & & & $418(21.0)$ \\
\hline "Values are no. (\%) unless otherwise indicated. CA-CO, community-acquired, community-onset; HCA-CO, healthcare-associated, community-onset; HO, \\
hospital-onset; ICU, intensive care unit.
\end{tabular}

and then remained stable $(23.7 \%$ in $2003-2007$ and $32.4 \%$ in 2008-2013, $\mathrm{p}<0.01)$. Fluoroquinolone-nonsusceptible isolates were more frequent in patients with healthcare exposure (community-acquired, community-onset $21.6 \%$; healthcare-associated, community-onset $37.6 \%$; hospitalonset $37.9 \%, p<0.01$ ), and nonsusceptibility rates increased in groups with these exposures (community-acquired, community-onset; healthcare-associated, community-onset; and hospital-onset, $\mathrm{p}<0.01$ by test for trend).

\section{Klebsiella spp.}

Carbapenem nonsusceptibility was reported for $3.0 \%$ of Klebsiella spp. isolates (Figure 2). We observed a trend toward increasing nonsusceptibility rates $(2.1 \%$ in $2003-$ 2007 and $3.7 \%$ in $2008-2013, \mathrm{p}<0.01$ by test for trend). Healthcare exposure was associated with higher rates of carbapenem nonsusceptibility (community-acquired, community-onset $0.9 \%$; healthcare-associated, community-onset $2.2 \%$; hospital-onset $5.6 \%, \mathrm{p}<0.01$ ).

Overall, $11.8 \%$ of isolates had nonsusceptible results to extended-spectrum cephalosporins and a relatively stable proportion of nonsusceptibility rates $(12.6 \%$ in $2003-$ 2007 and $11.2 \%$ in $2008-2013, p=0.07$ by test for trend). However, nonsusceptibility rates increased for communityacquired, community-onset isolates ( $p=0.04$ by test for trend) but not for healthcare-associated, community-onset $(\mathrm{p}=0.17)$ and hospital-onset isolates $(\mathrm{p}=0.21)$. Proportions that were nonsusceptible to extended-spectrum cephalosporins were more frequent for healthcare-associated, community-onset (10.4\%) and hospital-onset (20.2\%) isolates than for community-acquired, community-onset isolates $(3.9 \%, \mathrm{p}<0.01)$.

Overall, $9.8 \%$ of isolates were nonsusceptible to aminoglycosides, and a higher proportion were nonsusceptible for patients with healthcare exposure (community-acquired, community-onset $3.6 \%$; healthcare-associated, community-onset $8.5 \%$; hospital-onset $16.7 \%$, $\mathrm{p}<0.01)$. Overall, nonsusceptibility to aminoglycosides decreased $(10.9 \%$ in $2003-2007$ and $9.0 \%$ in 2008-2013, $\mathrm{p}<0.01$ by test for trend). However, nonsusceptibility increased for community-acquired, community-onset isolates $(2.9 \%$ in $2003-2007$ and $4.1 \%$ in $2008-2013$, p $=0.01$ by test for trend) and decreased for hospital-onset isolates (18.6\% in $2003-2007$ and $14.8 \%$ in $2008-2013$, $\mathrm{p}<0.01$ by test for trend).

Fluoroquinolone nonsusceptibility for all isolates was $12.6 \%$, and higher proportions were observed for patients with healthcare exposure (community-acquired, community-onset 5.3\%; healthcare-associated, community-onset $11.6 \%$; hospital-onset $20.1 \%, \mathrm{p}<0.01$ ). Among all isolates, the nonsusceptible proportion was stable during the study ( $p=0.12$ by test for trend). However, there were increasing nonsusceptibility rates for community-acquired, community-onset isolates $(4.7 \%$ in $2003-2007$ and $5.6 \%$ in 2008 $2013, p=0.04$ by test for trend), and there were no changes observed for other healthcare-associated, community-onset $(p=0.41)$ and hospital-onset $(p=0.28)$ isolates.

\section{P. aeruginosa}

Antipseudomonal carbapenem nonsusceptibility was reported for $15.8 \%$ of all isolates (Figure 3 ). The rates for antipseudomonal carbapenem-nonsusceptible isolates were stable during the study ( $p=0.66$ by test for trend). For community-acquired, community-onset isolates, the proportion of nonsusceptible isolates increased (4.8\% in 2003-2007 and $7.9 \%$ in $2008-2013, p=0.03$ by test for trend), but there was no significant trend for other categories (healthcare-associated, community-onset; $p=0.45$ and hospitalonset; $p=0.40$ ). Healthcare exposure was associated with higher rates of nonsusceptibility to antipseudomonal carbapenems (community-acquired, community-onset 6.6\%; healthcare-associated, community-onset $10.0 \%$; and hospital-onset $24.1 \%, \mathrm{p}<0.01$ ).

Healthcare exposure also increased nonsusceptibility to antipseudomonal extended-spectrum cephalosporins (community-acquired, community-onset 9.1\%; healthcareassociated, community-onset $15.5 \%$; hospital-onset $33.2 \%$, $\mathrm{p}<0.01$ ). Although $22.3 \%$ of all isolates were nonsusceptible, the proportion that were nonsusceptible decreased (24.8\% in $2003-2007$ and $20.0 \%$ in $2008-2013, \mathrm{p}<0.01$ 
by test for trend). Decreases were limited to healthcareexposed patients (healthcare-associated, community-onset; $p=0.01$ and hospital-onset; $p=0.04$, but not communityacquired, community-onset; $\mathrm{p}=0.16$ ).

The overall rate for aminoglycoside nonsusceptible isolates was $20.6 \%$, and nonsusceptible bacteria were more frequently isolated from patients with healthcare exposure (community-acquired, community-onset $10.7 \%$; healthcare-associated, community-onset $16.4 \%$; hospitalonset $28.0 \%, \mathrm{p}<0.01$ ). The overall trend was toward lower rates of nonsusceptible isolates $(24.1 \%$ in $2003-2007$ and $17.3 \%$ in $2008-2013, p<0.01$ by test for trend). However, this trend of decreasing aminoglycoside nonsusceptibility rates was significant only for patients with healthcare exposure (community-acquired, community-onset, $\mathrm{p}=0.14$; healthcare-associated, community-onset, $\mathrm{p}<0.01$; and hospital-onset, $\mathrm{p}<0.01$ ).

The overall rate for antipseudomonal fluoroquinolone-nonsusceptible isolates was $29.6 \%$; these isolates were obtained more frequently from patients who had healthcare-associated, community-onset $(24.6 \%)$ and hospital-onset episodes (39.4\%) than from patients who had community-acquired, community-onset episodes $(15.4 \%, \mathrm{p}<0.01)$. The proportion that was nonsusceptible decreased during the study $(32.2 \%$ in $2003-2007$ and $27.2 \%$ in $2008-2013, p<0.01$ by test for trend), but this trend was significant only for patients with healthcare exposure (community-associated, $\mathrm{p}=0.29$; healthcareassociated, community-onset, $\mathrm{p}<0.01$; and hospitalonset, $\mathrm{p}=0.02$ ).

\section{Acinetobacter spp.}

Antipseudomonal carbapenem nonsusceptibility results were reported for $9.8 \%$ of the isolates (Figure 4). The proportion of nonsusceptible isolates was higher for isolates from patients with healthcare exposure (community-acquired, community-onset 4.1\%; healthcare-associated, community-onset 9.4\%; and hospital-onset $32.6 \%$, $\mathrm{p}<0.01)$. The nonsusceptible proportion for hospital-onset isolates increased significantly from $15.0 \%$ in 2003 to $56.6 \%$ in 2009 , but decreased to $29.7 \%$ in 2013 (trend in 2003-2009, $\mathrm{p}<0.01$; trend in 2010-2013, $\mathrm{p}<0.01$ ). The nonsusceptibility proportion for community-acquired, community-onset isolates showed a significant trend $(\mathrm{p}=$
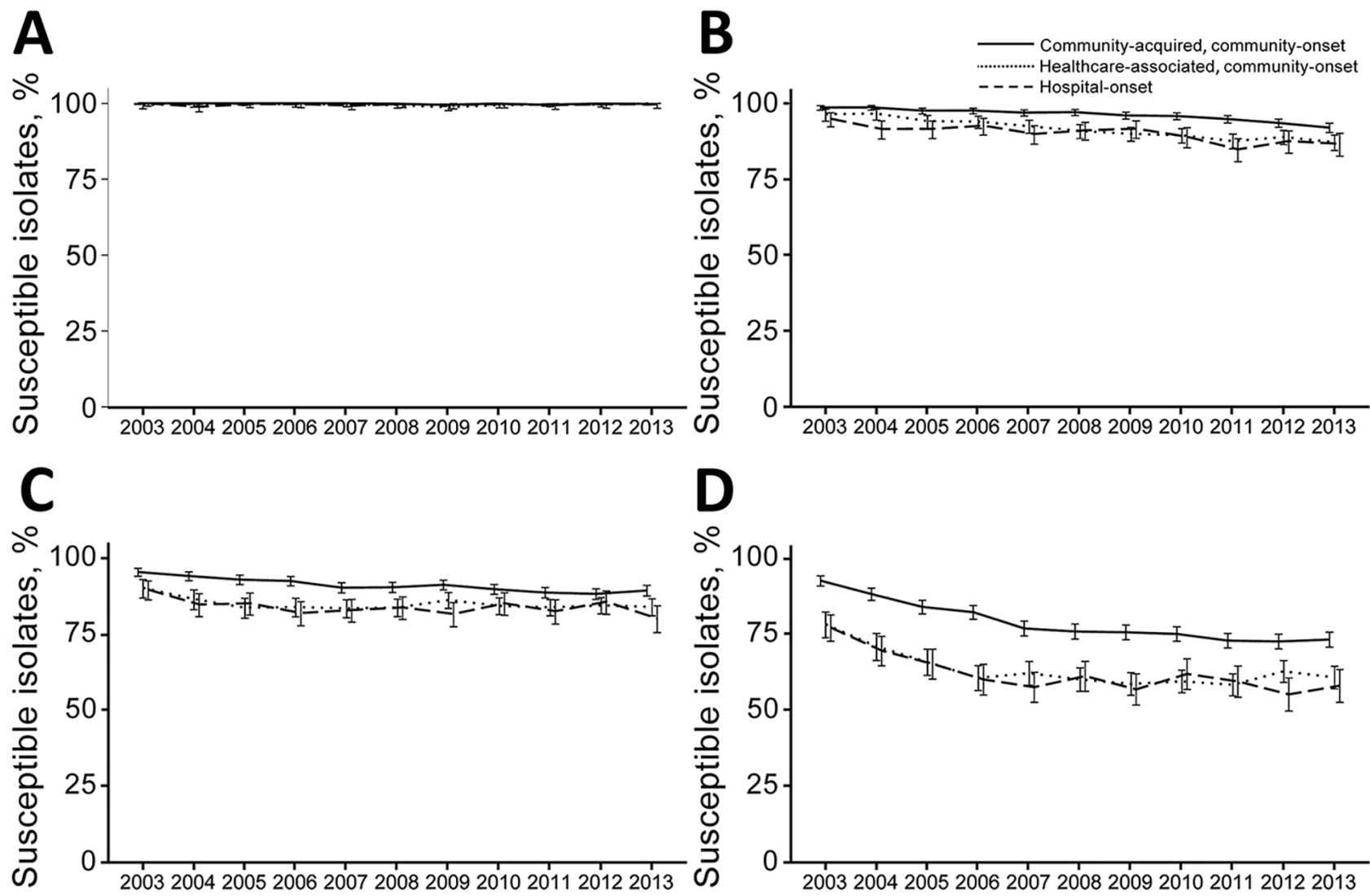

Figure 1. Trends of selected antimicrobial susceptibilities for Escherichia coli isolates from patients with bacteremia, Veterans Health Administration System, United States, 2003-2013. A) Carbapenems, B) Extended-spectrum cephalosporins, C) Aminoglycosides, D) Fluoroquinolones. Error bars indicate $95 \%$ Cls. 
0.41 ), but this trend increased for healthcare-associated, community-onset isolates (5.4\% in 2003-2007 and $12.1 \%$ in 2008-2013, $\mathrm{p}=0.04)$.

Overall, $48.1 \%$ of isolates were nonsusceptible to extended-spectrum cephalosporins, and this proportion decreased during the study (54.3\% in 2003-2007 and 42.5\% in 2008-2013, $\mathrm{p}<0.01$ by test for trend). This decrease was caused primarily by significant decreases in nonsusceptibility for hospital-onset isolates since 2009 (community-acquired, community-onset, $\mathrm{p}=0.28$; healthcare-associated, community-onset, $\mathrm{p}=0.23$; and hospital-onset, $\mathrm{p}<0.01)$. Extendedspectrum cephalosporin-nonsusceptible isolates were more frequent for patients with healthcare exposure (communityacquired, community-onset 19.6\%; healthcare-associated, community-onset $30.1 \%$; hospital-onset $70.4 \%$, p $<0.01$ ).

Although the overall proportion of aminoglycosidenonsusceptible isolates was $37.4 \%$, the proportion considered nonsusceptible was higher as healthcare exposure increased (community-acquired, community-onset 9.6\%; healthcare-associated, community-onset $19.9 \%$; and hospital-onset $60.0 \%, \mathrm{p}<0.01)$ The overall trend was toward decreasing rates of nonsusceptibility $(45.7 \%$ in $2003-2007$ and $29.9 \%$ in $2008-2013, \mathrm{p}<0.01$ by test for trend). The decrease was significant only for patients with healthcare exposure (community-acquired, community-onset, $\mathrm{p}=$ 0.08 ; healthcare-associated, community-onset, $\mathrm{p}=0.04$; and hospital-onset, $\mathrm{p}<0.01$ ).

The overall proportion of isolates that were nonsusceptible to fluoroquinolones was $47.2 \%$. Patients with healthcare exposure were more likely to be infected with nonsusceptible strains (community-acquired, community-onset 14.2\%; healthcare-associated, community-onset $19.6 \%$; hospitalonset $71.9 \%, \mathrm{p}<0.01)$ There were significant decreases in nonsusceptibility to fluoroquinolones (54.3\% in 2003-2007 and $41.0 \%$ in $2008-2013, \mathrm{p}<0.01$ by test for trend), which was observed for hospital-onset isolates only (communityacquired, community-onset, $\mathrm{p}=0.07$; healthcare-associated, community-onset, $\mathrm{p}=0.73$; hospital-onset, $\mathrm{p}<0.01$ ).

\section{Discussion}

We analyzed 47,746 cases of gram-negative bacteremia in the VHA system over an 11-year period and report 3 major findings. First, for E. coli, rates of nonsusceptibility to aminoglycosides, fluoroquinolones, and extended-spectrum cephalosporins increased across all care settings. Second, for Klebsiella spp., rates of carbapenem nonsusceptibility
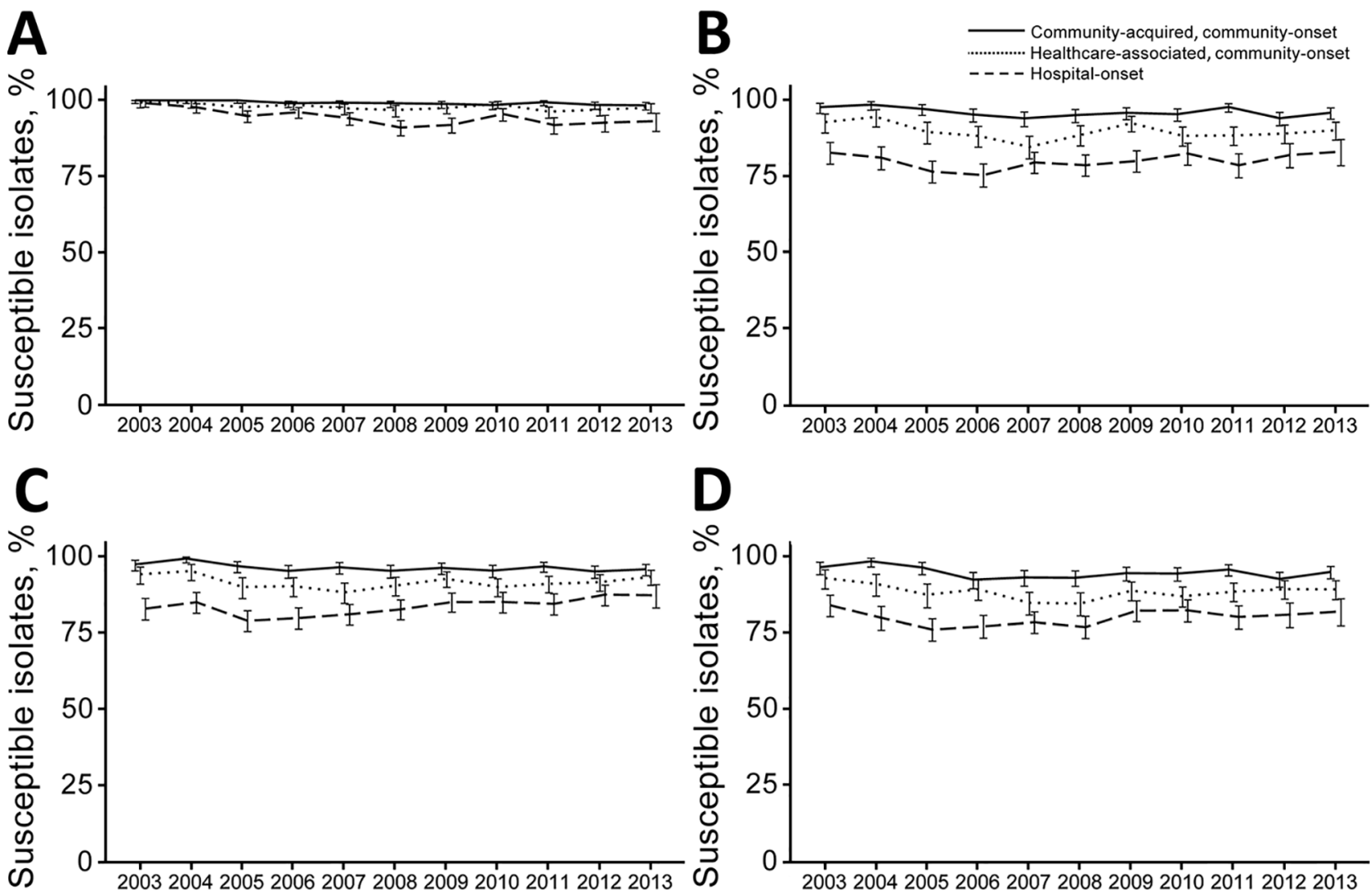

Figure 2. Trends of selected antimicrobial susceptibilities for Klebsiella spp. isolates from patients with bacteremia, Veterans Health Administration System, United States, 2003-2013. A) Carbapenems, B) Extended-spectrum cephalosporins, C) Aminoglycosides, D) Fluoroquinolones. Error bars indicate $95 \% \mathrm{Cls}$. 
increased across all care settings, and nonsusceptibility to other antimicrobial classes increased for community-onset isolates and was stable or decreased for isolates from patients with healthcare exposure. Third, for $P$. aeruginosa and Acinetobacter spp., rates of nonsusceptibility to aminoglycosides, fluoroquinolones, and extended-spectrum cephalosporins mostly decreased, but only for isolates from patients with healthcare exposure.

Overall incidence rates for gram-negative bacteremia were comparable with those from previous populationbased studies from the United States and Europe (3,13,20 22,30 ). We recently reported major changes in hospital-onset bacteremia, which was possibly caused by expansion of horizontal infection control programs in the VHA system during this time (15). An additional finding in our more recent study was the decreased proportion of nonsusceptible bloodstream isolates across nearly all antimicrobial classes for Klebsiella spp., P. aeruginosa, and Acinetobacter spp. in patients with healthcare exposure. However, trends for community-onset bloodstream isolates were stable or showed increased nonsusceptibility.

Our findings suggest that efforts to improve infection control practices within the VHA might have had effects on antimicrobial susceptibilities for isolates from patients with healthcare exposure, although the extent of these effects needs to be determined. With increased nonsusceptibility rates among community-acquired, community-onset isolates, we generally expect upward trends of nonsusceptibility rates for healthcare-associated and hospital-onset isolates caused by increased colonization pressure $(31,32)$. However, our results showed improvements in nonsusceptibility rates for isolates of Klebsiella spp., P. aeruginosa, and Acinetobacter spp. in hospital-onset and healthcare-associated, community-onset infections, which might suggest successful interruption of transmission in inpatient settings. Infection control programs within the VHA have been expanded over the past 10 years, especially since introduction of the Methicillin-Resistant Staphylococcus aureus Prevention Initiative in 2007 (33). Our recent study showed a substantial decrease in nosocomial, gram-negative bacteremia after implementation of this initiative, which suggests a collateral benefit beyond the originally intended scope of this initiative (15). In addition, the VHA released a mandatory policy for hand hygiene practices in 2011 (34).

Antimicrobial stewardship efforts within the VHA, which have been evolving over the past 10 years, might
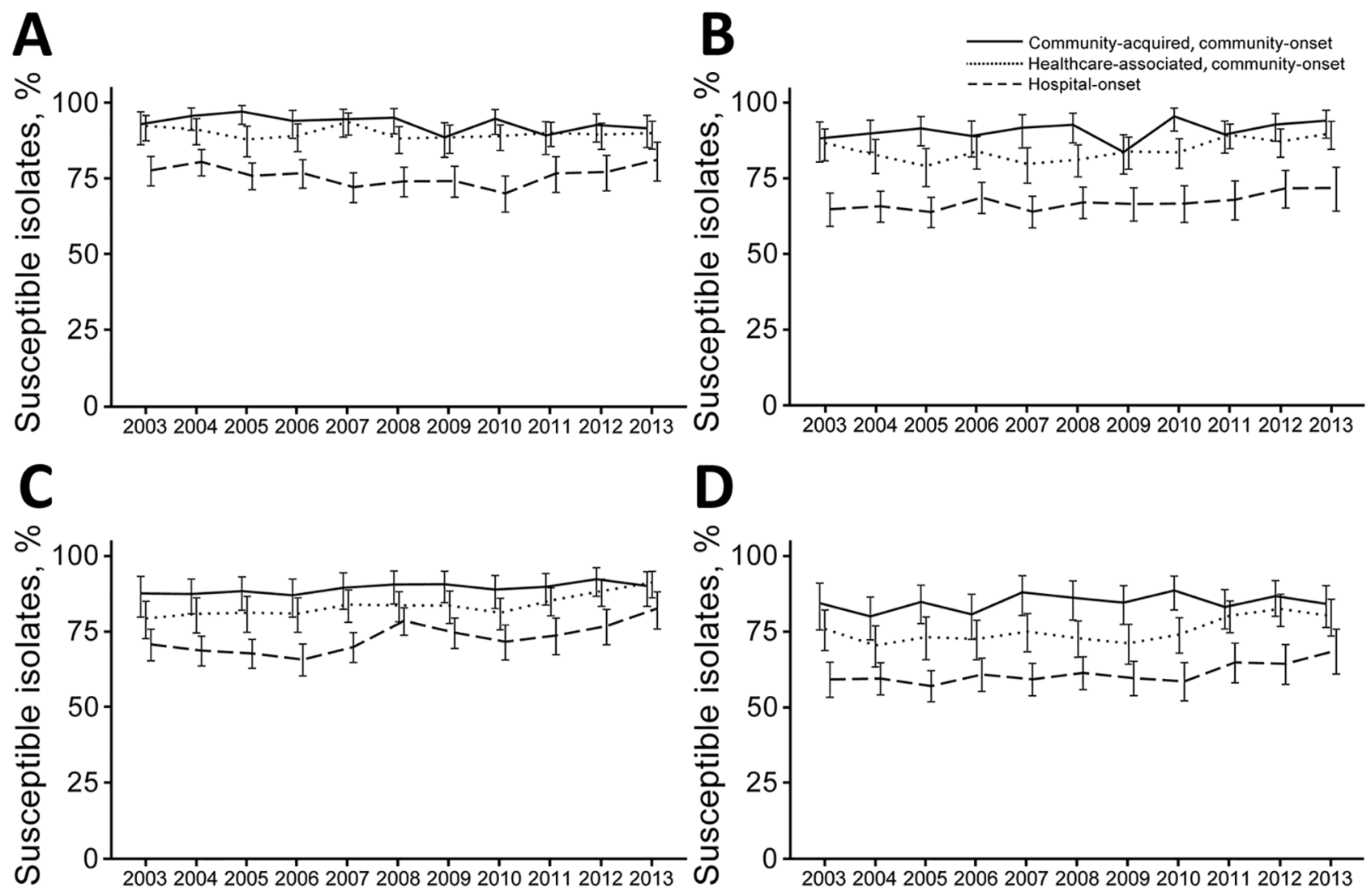

Figure 3. Trends of selected antimicrobial susceptibilities for Pseudomonas aeruginosa isolates from patients with bacteremia, Veterans Health Administration System, United States, 2003-2013. A) Antipseudomonal carbapenems, B) Antipseudomonal cephalosporins, C) Aminoglycosides, D) Antipseudomonal fluoroquinolones. Error bars indicate $95 \% \mathrm{Cls}$. 
have contributed to increased antimicrobial susceptibilities. The National VA Antimicrobial Stewardship Task Force, established in 2011, has been leading systemwide educational efforts, and a mandate for inpatient stewardship was enacted in 2014. However, even before these broad interventions, facilities were actively performing stewardship. According to a survey of VHA facilities conducted by the VA Healthcare Analysis and Information Group, 86 $(66.2 \%)$ of 130 VHA facilities had either a formal or informal antimicrobial stewardship policy in place in 2012 (35). At the time of the survey, 14 (16.3\%) of these facilities had a stewardship policy in place for 2 years, $7(8.1 \%)$ for 3 years, and $33(38.4 \%)$ for $\geq 4$ years.

We speculate that increased rates of antimicrobial susceptibility were not observed for $E$. coli healthcareassociated, community-onset and hospital-onset isolates because patients were probably colonized with $E$. coli before admission and these commensal strains might have developed or acquired resistance in the community setting. Antimicrobial drug resistance can develop in $E$. coli by its exposure to retail meat (36) and antimicrobial drug exposure outside VHA hospitals. Antimicrobial stewardship efforts within the VHA, like most healthcare systems, have typically not focused on outpatients. In addition, many non-VHA hospitals, which might have cared for patients in this cohort but were not captured by our analysis, did not have stewardship programs during this study $(37,38)$.

A notable exception to these trends is increased rates of carbapenem nonsusceptibility for Klebsiella spp. in community and hospital settings. The increase in carbapenem-resistant Klebsiella spp. was also reported outside the VHA (39-41). This observation deserves attention because it might indicate a lapse of infection control efforts at the VHA. The VHA implemented internal guidelines for carbapenem-resistant Enterobacteriaceae to address this issue in 2015, and additional research is needed to determine whether this intervention has had any positive effect on this major threat (42).

The strength of our study was inclusion of all bacteremia episodes from the entire VHA system, which enabled us to perform population-based analysis, including diverse care settings, for wide geographic regions. Our study demonstrated the advantage of an integrated health informatics infrastructure with a clinical data warehouse within the VHA system. To accurately monitor the burden and trends
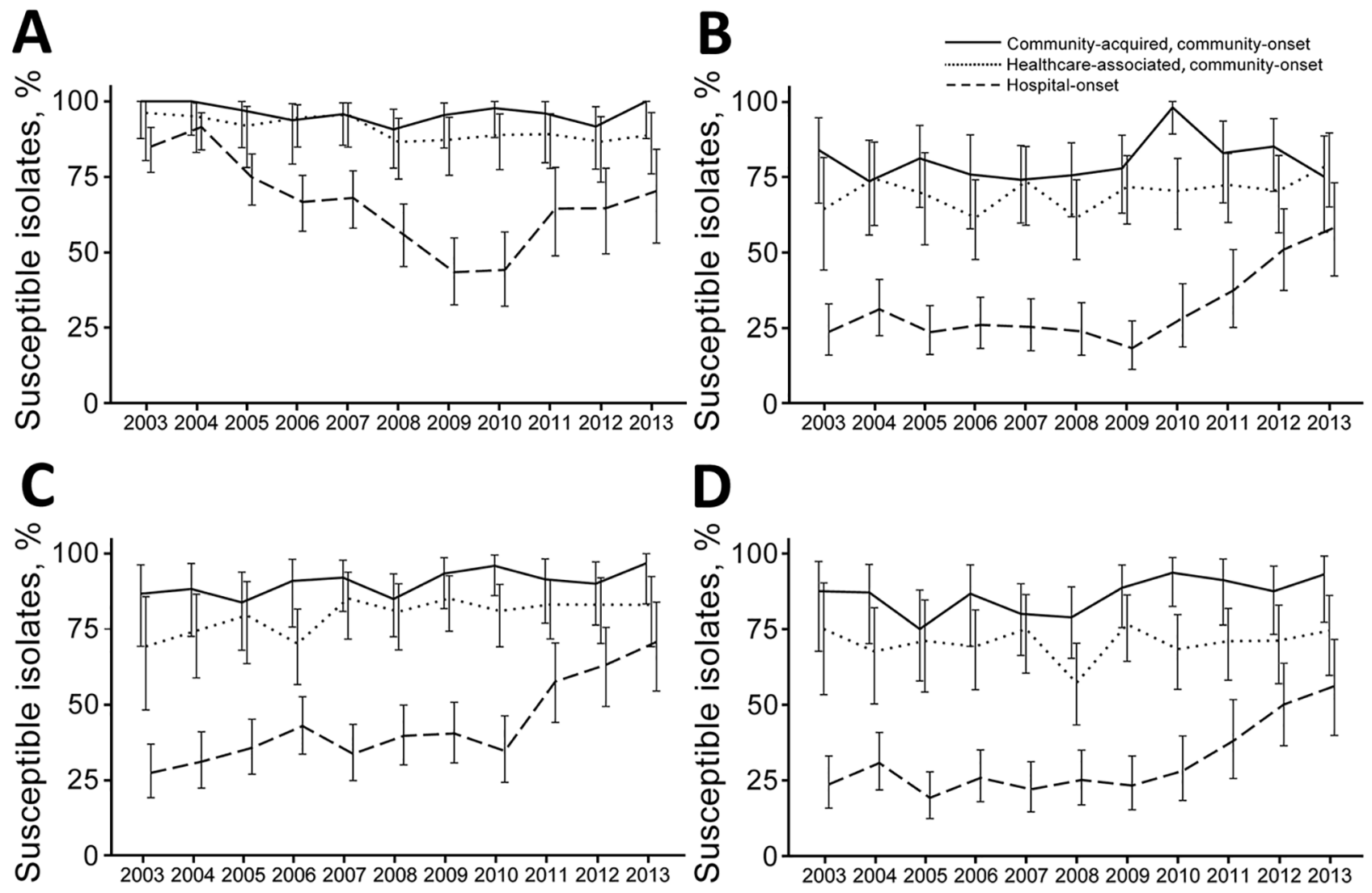

Figure 4. Trends of selected antimicrobial susceptibilities for Acinetobacter spp. isolates from patients with bacteremia, Veterans Health Administration System, United States, 2003-2013. A) Antipseudomonal carbapenems, B) Extended-spectrum cephalosporins, C) Aminoglycosides, D) Antipseudomonal fluoroquinolones. Error bars indicate 95\% Cls. 
of antimicrobial resistance, more investments in data integration informatics infrastructure need to be made.

This study had several limitations. First, most patients seen within the VHA system were male. Therefore, results might not be generalizable to the rest of the population. Second, because information was collected through patient medical records, detailed microbiological information, such as break points of antimicrobial susceptibility testing, molecular typing, or mechanisms of resistance, was often not available. Third, the Clinical and Laboratory Standards Institute (CLSI) revised its break point recommendations for extended-spectrum cephalosporins and carbapenems in 2010 (43), and antimicrobial susceptibility trends in the later part of the study might have been affected by this change. However, the FDA did not revise its recommendations to match those of the CLSI until 2013 (44). Because most microbiology laboratories in the VHA system use commercial systems that are regulated by the FDA for susceptibility testing, it is less likely that the change of CLSI break points substantially affected our observations. Fourth, this study focused on nationwide overall trends, and results might reflect local trends in each specific region. Previous studies showed geographic variabilities in incidence rates and antimicrobial susceptibilities for gram-negative bacteria $(45-47)$. We also recently reported regional variations in fluoroquinolone nonsusceptibilities for $E$. coli isolates and plan to analyze geographic variabilities for other organisms and antimicrobial agents in future studies (48). Fifth, resistance can develop in gram-negative bacteria during patient therapy, and assessing susceptibilities for the primary isolate from each episode might underestimate the prevalence of nonsusceptibilities. Because there were diversities in reporting practices of susceptibilities for subsequent isolates across the system, it was not feasible to collect information for those isolates in a standardized manner.

In conclusion, we observed an increase in antimicrobial drug nonsusceptibility for $E$. coli in all healthcare exposure categories and stable or decreased rates of nonsusceptibility for Klebsiella spp., P. aeruginosa, and Acinetobacter spp. in patients with healthcare exposure. The extent to which these findings might reflect VHA infection control and antimicrobial stewardship activities remains to be determined. Despite encouraging decreases in nonsusceptibility for pathogenic gram-negative bacteria in acute-care hospital settings, increased attention to infection prevention and antimicrobial stewardship is needed for outpatient and nonacute inpatient settings.

This study was supported by the Investigator Initiated Research program of Merck \& Co, Inc., and the Center for the Comprehensive Access and Delivery Research and Evaluation of the Iowa City Veterans Affairs Health Care System. E.N.P. was supported by a grant from Merck \& Co., Inc.

J.S.M. has received speaker honoraria from bioMérieux.
Dr. Goto is a network epidemiologist in the Veterans Administration Midwest Health Care Network (VISN 23) and clinical assistant professor of internal medicine at the University of Iowa Carver College of Medicine, Iowa City, IA. His research interests include epidemiology and prevention of multidrugresistant pathogens and biosurveillance.

\section{References}

1. Moreno R, Afonso S, Fevereiro T. Incidence of sepsis in hospitalized patients. Curr Infect Dis Rep. 2006;8:346-50. http://dx.doi.org/10.1007/s11908-006-0044-2

2. Goto M, Al-Hasan MN. Overall burden of bloodstream infection and nosocomial bloodstream infection in North America and Europe. Clin Microbiol Infect. 2013;19:501-9. http://dx.doi.org/10.1111/1469-0691.12195

3. Søgaard M, Nørgaard M, Dethlefsen C, Schønheyder HC. Temporal changes in the incidence and 30-day mortality associated with bacteremia in hospitalized patients from 1992 through 2006: a population-based cohort study. Clin Infect Dis. 2011;52:61-9. http://dx.doi.org/10.1093/cid/ciq069

4. Kang CI, Kim SH, Park WB, Lee KD, Kim HB, Kim EC, et al. Bloodstream infections caused by antibiotic-resistant gramnegative bacilli: risk factors for mortality and impact of inappropriate initial antimicrobial therapy on outcome. Antimicrob Agents Chemother. 2005;49:760-6. http://dx.doi.org/10.1128/ AAC.49.2.760-766.2005

5. Gikas A, Samonis G, Christidou A, Papadakis J, Kofteridis D, Tselentis Y, et al. Gram-negative bacteremia in non-neutropenic patients: a 3-year review. Infection. 1998;26:155-9. http://dx.doi.org/10.1007/BF02771841

6. Albrecht SJ, Fishman NO, Kitchen J, Nachamkin I, Bilker WB, Hoegg C, et al. Reemergence of gram-negative health careassociated bloodstream infections. Arch Intern Med. 2006;166:1289-94. http://dx.doi.org/10.1001/archinte. 166.12.1289

7. Luzzaro F, Ortisi G, Larosa M, Drago M, Brigante G, Gesu G. Prevalence and epidemiology of microbial pathogens causing bloodstream infections: results of the OASIS multicenter study. Diagn Microbiol Infect Dis. 2011;69:363-9. http://dx.doi.org/ 10.1016/j.diagmicrobio.2010.10.016

8. Prowle JR, Echeverri JE, Ligabo EV, Sherry N, Taori GC, Crozier TM, et al. Acquired bloodstream infection in the intensive care unit: incidence and attributable mortality. Crit Care. 2011;15:R100. http://dx.doi.org/10.1186/cc10114

9. Biedenbach DJ, Moet GJ, Jones RN. Occurrence and antimicrobial resistance pattern comparisons among bloodstream infection isolates from the SENTRY Antimicrobial Surveillance Program (1997-2002). Diagn Microbiol Infect Dis. 2004;50:59-69. http://dx.doi.org/10.1016/j.diagmicrobio.2004.05.003

10. Cordero L, Rau R, Taylor D, Ayers LW. Enteric gram-negative bacilli bloodstream infections: 17 years' experience in a neonatal intensive care unit. Am J Infect Control. 2004;32:189-95. http://dx.doi.org/10.1016/j.ajic.2003.07.004

11. Laupland KB. Defining the epidemiology of bloodstream infections: the 'gold standard' of population-based assessment. Epidemiol Infect. 2013;141:2149-57. http://dx.doi.org/10.1017/ S0950268812002725

12. Søgaard M, Lyytikäinen O, Laupland KB, Schønheyder HC. Monitoring the epidemiology of bloodstream infections: aims, methods and importance. Expert Rev Anti Infect Ther. 2013;11:1281-90. http://dx.doi.org/10.1586/14787210.2013.856262

13. Laupland KB. Incidence of bloodstream infection: a review of population-based studies. Clin Microbiol Infect. 2013;19:492-500. http://dx.doi.org/10.1111/1469-0691.12144 
14. Veterans Health Administration. About VHA, July 5, 2016 [cited 2017 Aug 29]. http://www.va.gov/health/aboutVHA.asp

15. Goto M, O'Shea AM, Livorsi DJ, McDanel JS, Jones MM, Richardson KK, et al. The effect of a nationwide infection control program expansion on hospital-onset gram-negative rod bacteremia in 130 Veterans Health Administration Medical Centers: an interrupted time-series analysis. Clin Infect Dis. 2016;63:642-50. http://dx.doi.org/10.1093/cid/ciw423

16. Shorr AF, Tabak YP, Killian AD, Gupta V, Liu LZ, Kollef MH. Healthcare-associated bloodstream infection: a distinct entity? Insights from a large U.S. database. Crit Care Med. 2006;34:258895. http://dx.doi.org/10.1097/01.CCM.0000239121.09533.09

17. Sligl W, Taylor G, Brindley PG. Five years of nosocomial gram-negative bacteremia in a general intensive care unit: epidemiology, antimicrobial susceptibility patterns, and outcomes. Int J Infect Dis. 2006;10:320-5. http://dx.doi.org/10.1016/ j.ijid.2005.07.003

18. Luzzaro F, Viganò EF, Fossati D, Grossi A, Sala A, Sturla C, et al.; AMCLI Lombardia Hospital Infectious Study Group. Prevalence and drug susceptibility of pathogens causing bloodstream infections in northern Italy: a two-year study in 16 hospitals. Eur J Clin Microbiol Infect Dis. 2002;21:849-55.

19. Friedman ND, Kaye KS, Stout JE, McGarry SA, Trivette SL, Briggs JP, et al. Health care-associated bloodstream infections in adults: a reason to change the accepted definition of communityacquired infections. Ann Intern Med. 2002;137:791-7. http://dx.doi.org/10.7326/0003-4819-137-10-200211190-00007

20. Al-Hasan MN, Eckel-Passow JE, Baddour LM. Impact of healthcareassociated acquisition on community-onset gram-negative bloodstream infection: a population-based study: healthcare-associated gram-negative BSI. Eur J Clin Microbiol Infect Dis. 2012;31:116371. http://dx.doi.org/10.1007/s10096-011-1424-6

21. Uslan DZ, Crane SJ, Steckelberg JM, Cockerill FR III, St Sauver JL, Wilson WR, et al. Age- and sex-associated trends in bloodstream infection: a population-based study in Olmsted County, Minnesota. Arch Intern Med. 2007;167:834-9. http://dx.doi.org/10.1001/archinte.167.8.834

22. Rodríguez-Créixems M, Alcalá L, Muñoz P, Cercenado E, Vicente T, Bouza E. Bloodstream infections: evolution and trends in the microbiology workload, incidence, and etiology, 1985-2006. Medicine (Baltimore). 2008;87:234-49. http://dx.doi.org/10.1097/ MD.0b013e318182119b

23. US Census Bureau. Our nation's veterans. Distribution of civilian veterans, 18 years and over in the United States and Puerto Rico, July 29, 2017 [cited 2017 Aug 29]. https://www.census.gov/library/ visualizations/2015/comm/our-nation-s-veterans.html

24. VHA. VHA Office of Rural Health, July 5, 2016 [cited 2017 Aug 29]. http://www.ruralhealth.va.gov/about/rural-veterans.asp

25. VHA. Facts about VA health care capabilities, July 5, 2016 [cited 2017 Aug 29]. http://www.publichealth.va.gov/docs/flu/pandemic/ AppxA3_HealthCare.pdf

26. Horan TC, Andrus M, Dudeck MA. CDC/NHSN surveillance definition of health care-associated infection and criteria for specific types of infections in the acute care setting. Am J Infect Control. 2008;36:309-32. http://dx.doi.org/10.1016/ j.ajic.2008.03.002

27. Magiorakos AP, Srinivasan A, Carey RB, Carmeli Y, Falagas ME, Giske CG, et al. Multidrug-resistant, extensively drug-resistant and pandrug-resistant bacteria: an international expert proposal for interim standard definitions for acquired resistance. Clin Microbiol Infect. 2012;18:268-81. http://dx.doi.org/10.1111/ j.1469-0691.2011.03570.x

28. Centers for Disease Control and Prevention. Age standardization and population estimates, July 27, 2017 [cited 2017 Aug 29]. https:/www.cdc.gov/nchs/tutorials/NHANES/NHANESAnalyses/ agestandardization/age_standardization_intro.htm
29. Clopper CJ, Pearson ES. The use of confidence or fiducial limits illustrated in the case of the binomial. Biometrika. 1934;26:404-13. http://dx.doi.org/10.1093/biomet/26.4.404

30. Skogberg K, Lyytikäinen O, Ollgren J, Nuorti JP, Ruutu P. Population-based burden of bloodstream infections in Finland. Clin Microbiol Infect. 2012;18:E170-6. http://dx.doi.org/10.1111/ j.1469-0691.2012.03845.x

31. Bonten MJ, Slaughter S, Ambergen AW, Hayden MK, van Voorhis J, Nathan C, et al. The role of "colonization pressure" in the spread of vancomycin-resistant enterococci: an important infection control variable. Arch Intern Med. 1998;158:1127-32. http://dx.doi.org/10.1001/archinte.158.10.1127

32. Bonten MJ, Gaillard CA, Johanson WG Jr, van Tiel FH, Smeets HG, van der Geest S, et al. Colonization in patients receiving and not receiving topical antimicrobial prophylaxis. Am J Respir Crit Care Med. 1994;150:1332-40. http://dx.doi.org/ 10.1164/ajrccm.150.5.7952561

33. Jain R, Kralovic SM, Evans ME, Ambrose M, Simbartl LA, Obrosky DS, et al. Veterans Affairs initiative to prevent methicillin-resistant Staphylococcus aureus infections. N Engl J Med. 2011;364:1419-30. http://dx.doi.org/10.1056/ NEJMoa1007474

34. VHA. VHA directive 2011-007. Required hand hygiene practices, July 5, 2016 [cited 2017 Aug 29]. http://www1.va.gov/ vhapublications/ViewPublication.asp?pub_ID=2367

35. Chou AF, Graber CJ, Jones M, Zhang Y, Goetz MB, Madaras-Kelly K, et al. Characteristics of antimicrobial stewardship programs at Veterans Affairs hospitals: results of a nationwide survey. Infect Control Hosp Epidemiol. 2016; 37:647-54. http://dx.doi.org/10.1017/ice.2016.26

36. Overdevest I, Willemsen I, Rijnsburger M, Eustace A, Xu L, Hawkey P, et al. Extended-spectrum $\beta$-lactamase genes of Escherichia coli in chicken meat and humans, the Netherlands. Emerg Infect Dis. 2011;17:1216-22. http://dx.doi.org/10.3201/ eid1707.110209

37. Pogorzelska-Maziarz M, Herzig CT, Larson EL, Furuya EY, Perencevich EN, Stone PW. Implementation of antimicrobial stewardship policies in U.S. hospitals: findings from a national survey. Infect Control Hosp Epidemiol. 2015;36:261-4. http://dx.doi.org/10.1017/ice.2014.50

38. Centers for Disease Control and Prevention. Percent of hospitals with antibiotic stewardship programs by state, 2014. July 5, 2016 [cited 2017 Aug 29]. http://www.cdc.gov/getsmart/community/ pdfs/stewardship_11_13.pdf

39. Sievert DM, Ricks P, Edwards JR, Schneider A, Patel J, Srinivasan A, et al.; National Healthcare Safety Network (NHSN) Team and participating NHSN facilities. Antimicrobial-resistant pathogens associated with healthcare-associated infections: summary of data reported to the National Healthcare Safety Network at the Centers for Disease Control and Prevention, 2009-2010. Infect Control Hosp Epidemiol. 2013;34:1-14. http://dx.doi.org/10.1086/668770

40. Centers for Disease Control and Prevention. Tracking CRE, July 5, 2016 [cited 2017 Aug 29]. http://www.cdc.gov/hai/organisms/cre/ TrackingCRE.html

41. Thaden JT, Lewis SS, Hazen KC, Huslage K, Fowler VG Jr, Moehring RW, et al. Rising rates of carbapenem-resistant enterobacteriaceae in community hospitals: a mixed-methods review of epidemiology and microbiology practices in a network of community hospitals in the southeastern United States. Infect Control Hosp Epidemiol. 2014;35:978-83. http://dx.doi.org/10.1086/677157

42. Veterans Health Administration. VHA guideline for control of carbapenemase-producing carbapenem-resistant Enterobacteriaceae (CP-CRE). Washington (DC): MDRO Prevention Office, National Infectious Diseases Service, The Administration; 2015. 
43. Clinical Laboratory Standards Institute. Performance standards for antimicrobial susceptibility testing; 21 st informational supplement. Document M100-S21. Wayne (PA). The Institute; 2010.

44. Food and Drug Administration. Antibacterial and antifungal product labeling: microbiology susceptibility interpretive criteria (breakpoints) and quality control parameter updates, July 5, 2016 [cited 2017 Aug 29]. http://www.fda.gov/AboutFDA/Centers Offices/OfficeofMedicalProductsandTobacco/CDER/ucm275763.htm

45. Fisman D, Patrozou E, Carmeli Y, Perencevich E, Tuite AR, Mermel LA; Geographical Variability of Bacteremia Study Group. Geographical variability in the likelihood of bloodstream infections due to gram-negative bacteria: correlation with proximity to the equator and health care expenditure. PLoS One. 2014;9:e114548. http://dx.doi.org/10.1371/journal.pone.0114548

46. Hawkey PM, Jones AM. The changing epidemiology of resistance. J Antimicrob Chemother. 2009;64(Suppl 1):i3-10. http://dx.doi.org/10.1093/jac/dkp256
47. Wilson BM, El Chakhtoura NG, Patel S, Saade E, Donskey CJ, Bonomo RA, et al. Carbapenem-resistant Enterobacter cloacae in patients from the US Veterans Health Administration, 2006-2015. Emerg Infect Dis. 2017;23:878-80. http://dx.doi.org/10.3201/ eid2305.162034

48. Livorsi DJ, Goto M, Carrel M, Jones MM, McDanel J, Nair R, et al. Regional variations in fluoroquinolone nonsusceptibility among Escherichia coli bloodstream infections within the Veterans Healthcare Administration. Antimicrob Resist Infect Control. 2016;5:38. http://dx.doi.org/10.1186/ s13756-016-0135-2

Address for correspondence: Michihiko Goto, Iowa City Veterans Affairs Health Care System, University of Iowa Carver College of Medicine, 601 Hwy 6 W, Iowa City, IA 52246, USA; email: michihiko-goto@uiowa.edu

\section{The Public Health Image Library (PHIL)}

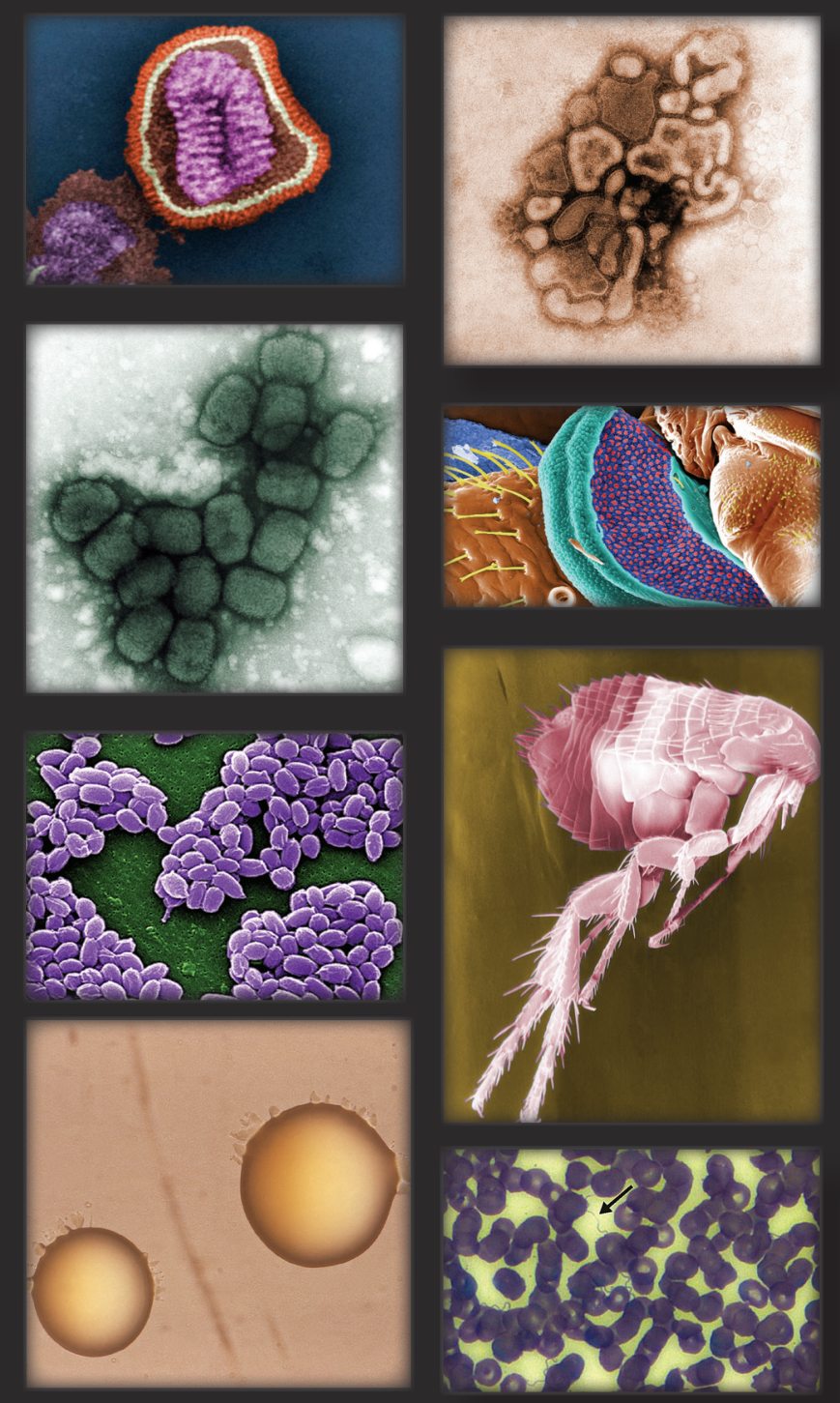

The Public Health Image Library

(PHIL), Centers for Disease Control and Prevention, contains thousands of public health-related images, including highresolution (print quality) photographs, illustrations, and videos.

PHIL collections illustrate current events and articles, supply visual content for health promotion brochures, document the effects of disease, and enhance instructional media.

PHIL images, accessible to PC and Macintosh users, are in the public domain and available without charge.

\section{Visit PHIL at:}

\section{http: / /phil.cdc.gov/phil}

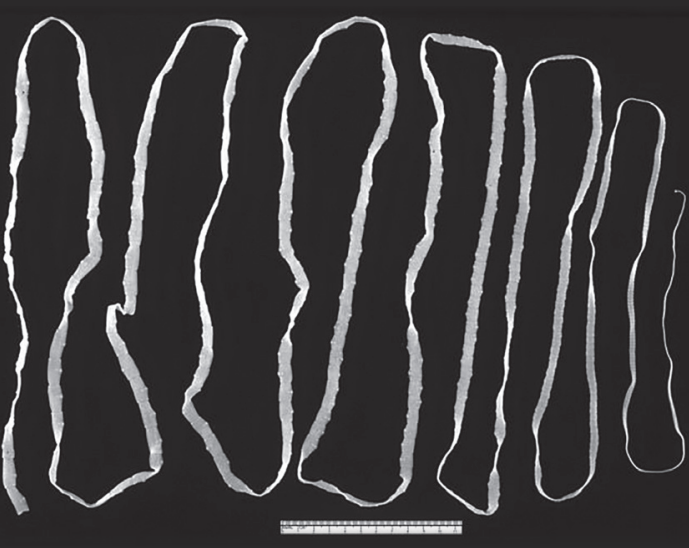

